\title{
An in situ measurement of flow field of river based on four-rotor UAV
}

\author{
ZHANG $\mathrm{yu}^{1, \mathrm{a}} \quad$ JIANG Qinghui ${ }^{2, \mathrm{~b}}$ \\ 1.Yangtze River Scientific Research Institute, Wuhan 430010, China \\ 2.School of Civil Engineering Wuhan University, Wuhan 430072,China \\ a.zhangyu_1999@126.com \\ b. 29372440@qq.com
}

Keywords: Unmanned aerial vehicle (UAV), Flow field, Multi-scale, Optical flow

Abstract: An in situ measurement method of flow field by four-rotor unmanned aerial vehicle (UAV) is proposed, which is mainly based on the following considerations: (1) Four-rotor UAV is a highly integrated image acquisition tool; (2) The operation is easy and the price is cheap; (3) It has good adaptability to complex environment. A multi-scale optical flow analysis method is developed to estimate the in-situ flow field of the river surface. Experiment results show that under certain conditions, the four-rotor unmanned aerial vehicle can achieve in situ river surface flow field measurement.

\section{Introduction}

Particle Image Velocimetry (PIV) is an important part of experimental fluid mechanics [1]. It can be used to measure the velocity and flow pattern of instantaneous full-field surface such as river course and water conservancy project. Compared with the velocity meter, the image analysis method based on PIV is mainly used to measure the surface of the fluid. It has the advantages of wide measuring range and relatively convenient operation. However, its flexibility, convenience and accuracy are limited by the conditions of field lighting, image collection, calculation and analysis.

In this paper, an in situ measurement method of flow field by four-rotor UAV is proposed, which is mainly based on the following considerations: (1) Four-rotor UAV is a highly integrated image acquisition tool; (2) The operation is easy and the price is cheap; (3)It has good adaptability to complex environment [2]. A multi-scale optical flow analysis method is developed to estimate the in-situ flow field of a river channel. Experiment results show that under certain conditions, the four-rotor unmanned aerial vehicle can achieve in situ river surface flow field measurement.

\section{UAV data acquisition}

The Phantom 3 UAV of DJI-Innovations Company is used to study the method. It is a small four-rotor UAV, which consists of racks, flight control (including GPS and IMU), PTZ, single lens camera, HD video surveillance system and ground remote control. Its flight performance is stable, the shooting effect is good and the price is reasonable, conforms to the field in situ test the demand.

The aim of the altitude control is to control the photographic object distance so as to ensure the desired ground sampling distance (GSD) and sample range. According to the aerial photogrammetry knowledge may obtain the photography object distance formula [3](see Eq.(1)). 


$$
\begin{aligned}
& \text { PixelSize }=\frac{\text { Sensorwidth }}{\text { ImageWidth }} . \\
& G S D=\frac{\text { PixelSize } \times \text { ElevationAboveGround }}{\text { FocalLength }} .
\end{aligned}
$$

A simple calculation shows that when the four-rotor UAV hover over the river channel at a height of $50 \mathrm{~m}$, it covers a range of about $100 \mathrm{~m}$ long and $70 \mathrm{~m}$ wide, and the GSD of video is 2.5 $\mathrm{cm}$. In most cases, this resolution can be used to identify the natural markers floating on the surfaces of small and medium-sized natural river courses, such as foliage, foam, garbage and other floating material. The data acquisition of the flow field by UAV is shown in Fig.1 (a). The UAV is located above the river. The height of the UAV above the surface of the river is relative height. When the UAV took off from the river bank, the relative height can be considered equal to the object distance, or must provide elevation offset. Since the relative height is provided by the Phantom 3 control system, the object distance can be calculated by the relative height of UAV and its take-off point. The GSD can be calculated according to equation (1), and then the displacement of the image pixels on the video can be converted to the actual spatial displacement. Combined with the sampling frequency of the video, the velocity and the flow pattern of the measured surface can be obtained. Actual video sample data is shown in Fig.1 (b), for the Lishui River in Hubei Xinzhou, shooting for the state of the water under the bridge. As the shooting time is in the flood season, the river surface velocity is faster. The foam and leaves on the river form natural tracer markers. UAV is located 10 meters above the bridge, about 15 meters from the surface of the river and the GSD is $0.75 \mathrm{~cm}$. Through a simple calculation shows that the video covers a range of about $15 \mathrm{X} 15$ meters, the water flow velocity of about $1.5 \mathrm{~m} / \mathrm{s}$. UAV drift in the air positioning, in less wind conditions, the horizontal drift speed is generally less than $10 \mathrm{~cm} / \mathrm{s}$, resulting in video image background offset relative error of less than $10 \%$. However, if the wind speed has a great impact, the UAV will swing in the air, and then the video image background shift is larger, it must be processed. Fig.1 (c) shows the displacement of the fixed position in the manually sampled video scene, which represents the background movement caused by unmanned aerial vehicles swinging or drifting. Considering the elevation difference between the background and the height of the velocity field, the velocity field is corrected by the statistical data to form the corrected actual flow field, as shown in Fig.1 (d).

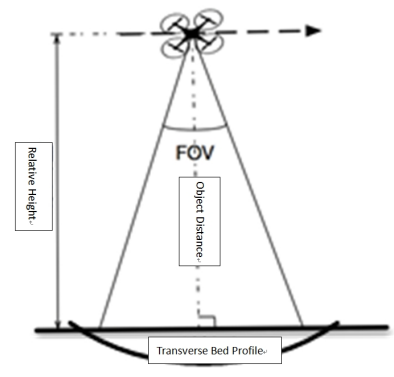

(a)

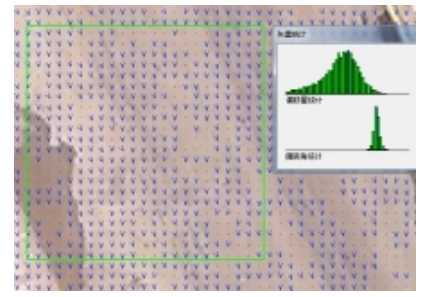

(c)

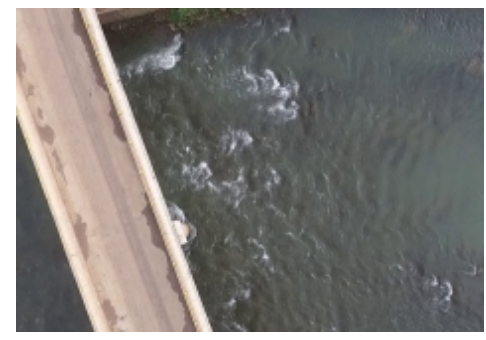

(b)

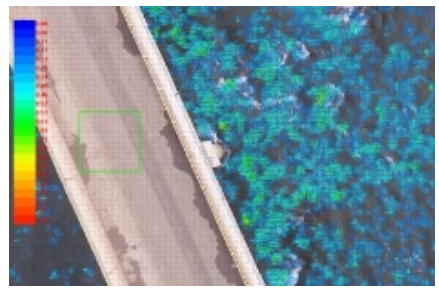

(d)

Fig.1. Schematic Diagram 
The process of in situ surface flow field estimation based on the UAV is shown in Fig.2. First, select the appropriate surface conditions and weather conditions to measure. In general, it is required that the surface of natural water body has a lot of floating objects as the tracing mark, otherwise the velocity of image can not be perceived. The scene must avoid the presence of large wind speed, so as not to cause instability in hover. Video content need to cover some fixed backgrounds, such as the shore, bridge and other buildings, as the fixed point in the video to calculate the amount of background movement caused by the hover instability of the UAV. Phantom 3 provides 4096x2160 resolution and 25 frames per second video, and can continue shooting about 20 minutes. The surface flow field can be calculated by selecting some video segment.

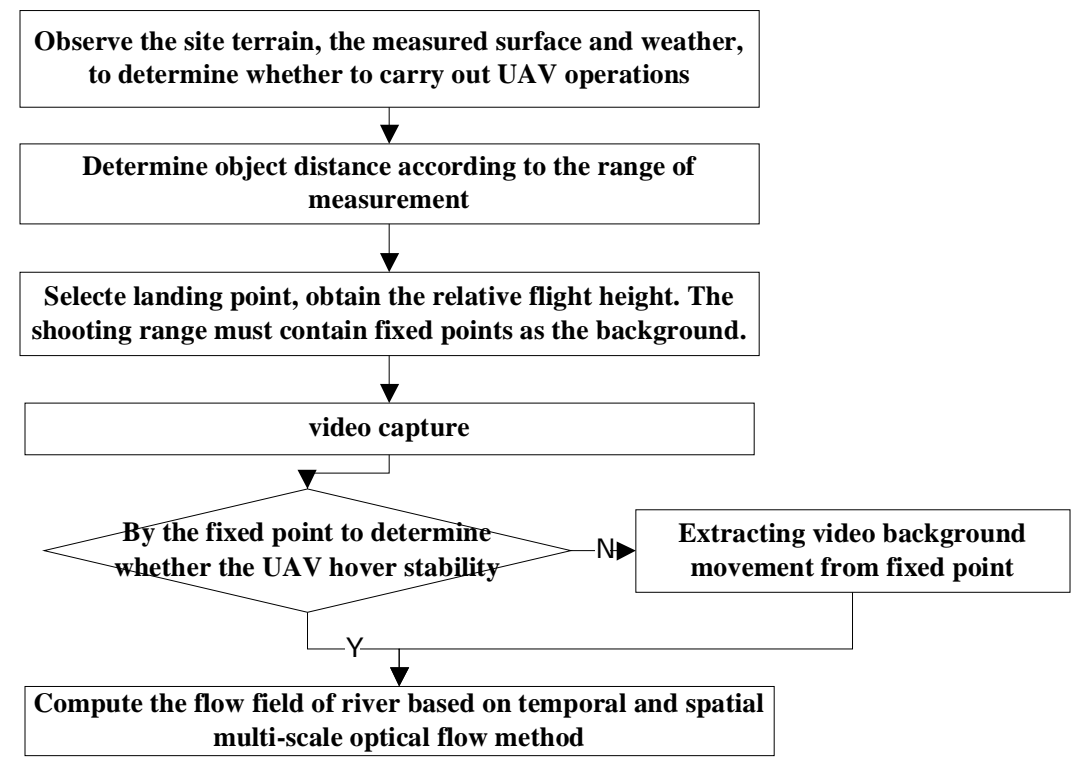

Fig.2. Work Flow

\section{Calculation of temporal and spatial multi-scale optical flow}

The mutual correlation principle is used in the velocity measurement of target motion based on sequence images. However, cross-correlation surface displacement measurements often introduce errors due to the presence of some erroneous matching. A basic assumption of the filtering approach is that the surface displacement is continuous, i.e., the displacement field is a relatively smooth field. Another approach is the use of optical flow for surface displacement analysis. In the PIV system, the cross-correlation means that the intensity of the same image point does not change with time, and the surface displacement of the fluid is generally a smooth value, that is, the velocity change of adjacent points in flow field is small. This results in two basic constraints of the optical flow [4] as shown in Eq.(2).

$$
\begin{aligned}
& \frac{\partial I}{\partial x} u+\frac{\partial I}{\partial y} v+\frac{\partial I}{\partial t}=0 . \\
& \left(u-u_{c}\right)^{2}+\left(v-v_{c}\right)^{2}=0 .
\end{aligned}
$$

Where $I$ is the image intensity, $(u, v)$ is the velocity vector of current point, $\left(u_{c}, v_{c}\right)$ is the neighborhood mean of the current point velocity. There are many ways to calculate the optical flow, and with many variations in the smoothness constraint. Calculating the optical flow must also keep the displacement of the two adjacent frames tiny, which causes the larger displacement to be undetectable. In general, natural river flow rate of 0.5-10 m / s, in Figure 1 (b) as an example, when 
the water flow rate of $1.5 \mathrm{~m} / \mathrm{s}$, the inter-frame pixel displacement of $6 \mathrm{~cm}$, or 8 pixels. If the flow rate is greater than $10 \mathrm{~m} / \mathrm{s}$, the inter-frame pixel displacement is greater than 53 pixels, if less than $0.5 \mathrm{~m} / \mathrm{s}$, the inter-frame pixel displacement is less than 2.7 pixels. It can be seen that when the flow regime are complex, such as the mountain river channel, the inter-frame pixel displacement will be larger than the effective calculation range of optical flow. Although the spatial multi-scale method is adopted, it is still impossible to calculate the large displacement, while the displacement is too small and reduce the displacement calculation accuracy. Therefore, the unreasonable displacement will lead to the error of optical flow calculation. Therefore, the disordered displacement will lead to optical flow calculation error, we must adjust the frame sampling interval to obtain a more appropriate pixel displacement to calculate optical flow.

Spatial and temporal multi scale optical flow calculation process is shown in Fig.3. Assuming that the flow field keeps constant in a short period of time, video by the UAV is analyzed with different frame sampling. High frame sampling frequency is used to analyze the fast flow field and low frequencies for analysis Low-speed flow field, and the final result is their effective fusion.

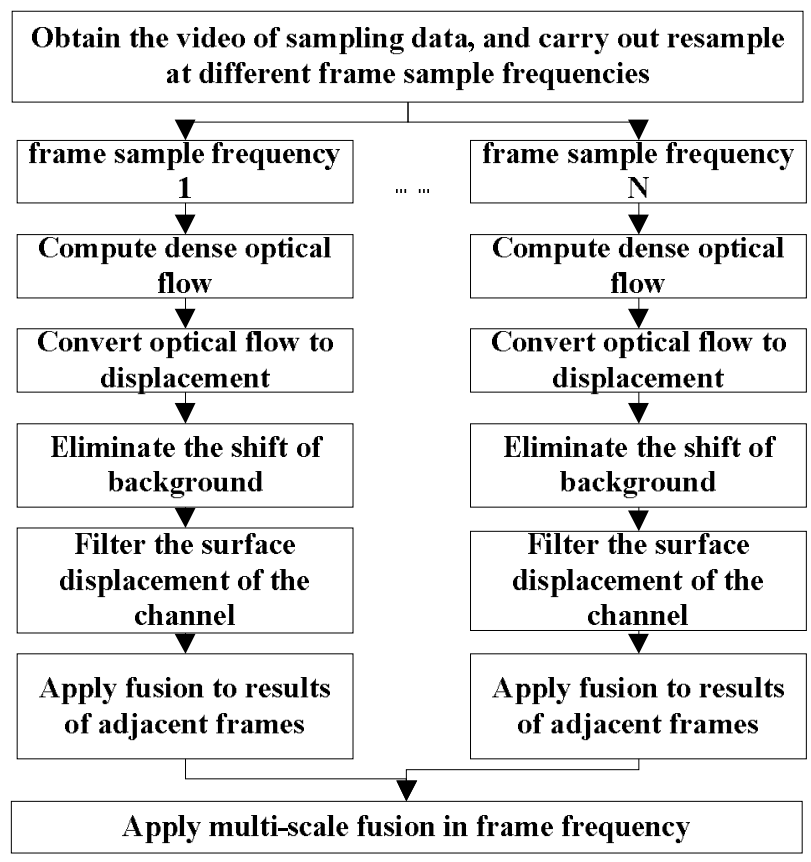

Fig.3.Spatial and temporal multi scale optical flow calculation

Fig.4 (a) shows a result of video image analysis with low frequency frame sampling. Fig.4 (b) is the one of high frequency frame analysis. The final fusion is shown in Fig.4 (c). The basic condition of this flow velocity fusion is assumed to be constant within 1 second. Video sampling frequencies are 10 and 2 respectively in flow field calculation. By fusion the results of multi-frame video image analysis at different frame sampling frequencies in a short time, the measurement of flow field with large change in the same scene is achieved.

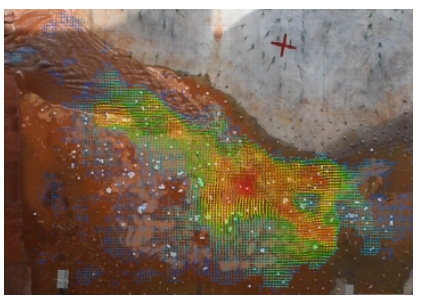

(a)

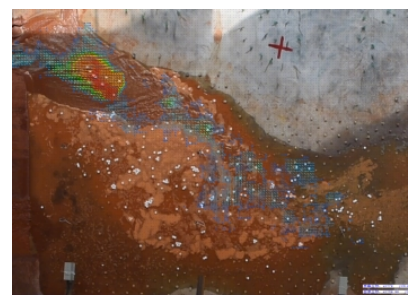

(b)

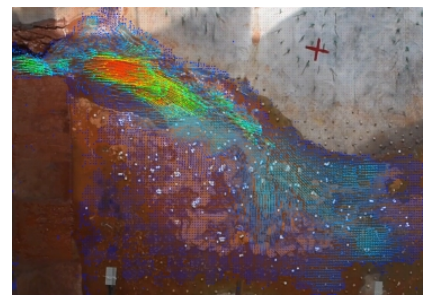

(c)

Fig.4. The fusion of optical flow calculation results at different frame sampling frequencies 


\section{Experimental results and conclusions}

Fig.5 shows the flow field of the Jiulong River in Qujing City, Yunnan Province. UAV hover over the river, about 30 meters high from the surface of the river. The wind speed is $2 \mathrm{~m} / \mathrm{s}$. Due to the drift of the GPS, the UAV swings slightly. The swing range is less than 30 pixels in video. The moving speed of the background is less than 1 pixel per second. The velocity of the river is more than 20 pixels per second, so the effect of background movement can be ignored. Fig.5 (a) shows the flow field calculated from the UAV video, and the result is superimposed on the video image in the form of the arrow vector. It can be seen that the velocity information can be obtained accurately according to the change of river surface texture, such as the place of wave rolling in the river. Otherwise, reliable speed information can not be obtained without such texture. Fig.5 (b) for the flow rate rendering. Above tests show that under certain conditions, the four-rotor UAV can achieve river surface flow field in situ measurement.

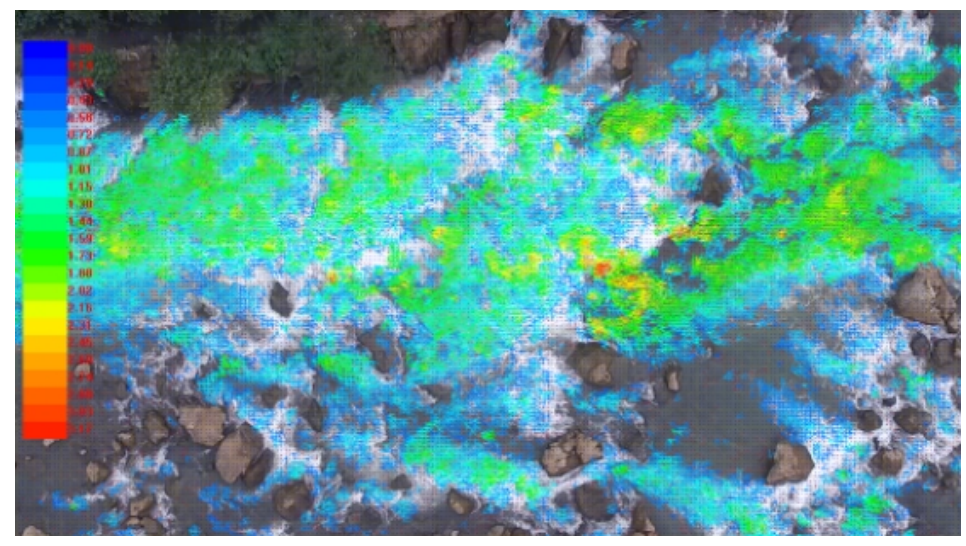

(a)

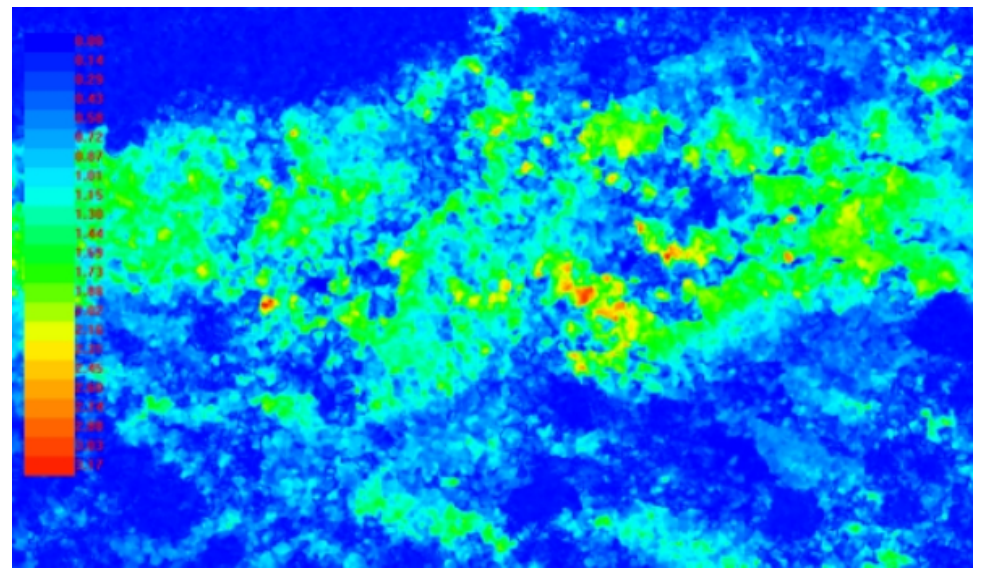

(b)

Fig.5. The calculation result of the flow field obtained by four-rotor UAV

\section{References}

[1]Ding Lihua, Zhang Xiaoguang, Zhao Liang, Zhang Haiming, Application of PIV Digital Image Testing Technology in TunDish Water Model Experiment[C], 2011 CSM Annual Meeting Proceedings ,2011.

[2] Ichiro Fujita, ,Yohei Kunita Application of aerial LSPIV to the 2002 flood of the Yodo River using a helicopter mounted high density video camera. Journal of Hydro-environment Research. Volume 5, Issue 4, December 2011, Pages 323-331. 
[3] Abdulla Al-Rawabdeh, Fangning He, Adel Moussa, Naser El-Sheimy and Ayman Habib, Using an Unmanned Aerial Vehicle-Based Digital Imaging System to Derive a 3D Point Cloud for Landslide Scarp Recognition. Remote Sens. 2016, 8(2), 95.

[4] Gunnar Farneback, Two-frame motion estimation based on polynomial expansion, Lecture Notes in Computer Science, 2003, (2749), 363-370. 